\title{
Extruding Plastic Scintillator at Fermilab
}

\author{
Anna Pla-Dalmau, Alan D. Bross, Victor V. Rykalin
}

\begin{abstract}
An understanding of the costs involved in the production of plastic scintillators and the development of a less expensive material have become necessary with the prospects of building very large plastic scintillation detectors. Several factors contribute to the high cost of plastic scintillating sheets, but the principal reason is the labor-intensive nature of the manufacturing process. In order to significantly lower the costs, the current casting procedures had to be abandoned. Since polystyrene is widely used in the consumer industry, the logical path was to investigate the extrusion of commercial-grade polystyrene pellets with dopants to yield high quality plastic scintillator. This concept was tested and high quality extruded plastic scintillator was produced. The Do and MINOS experiments are already using extruded scintillator strips in their detectors. An extrusion line has recently been installed at Fermilab in collaboration with NICADD (Northern Illinois Center for Accelerator and Detector Development). This new facility will serve to further develop and improve extruded plastic scintillator. This paper will discuss the characteristics of extruded plastic scintillator and its raw materials, the different manufacturing techniques and the current $R \& D$ program at Fermilab.
\end{abstract}

\section{INTRODUCTION}

XTRUDED plastic scintillators have become of interest in
these past few years as the need for building large plastic scintillation detectors has materialized. Cast plastic scintillator sheets may cost about $\$ 40$ per $\mathrm{kg}$. At this price, the plastic scintillator for a large detector is not affordable. However, using extruded plastic scintillator, the MINOS experiment was able to build two calorimeters, requiring almost 300 tons of finished plastic scintillator [1]. The overall cost of the extruded plastic scintillator for MINOS was calculated at about $\$ 10$ per $\mathrm{kg}$. A better understanding and optimization of the extrusion process and quality control procedures will result

This work was supported by the U.S. Department of Energy under Contract No. DE-AC02-76CHO3000 and in part by the U.S. Department of Education under Grant No. P116Z010035, the U.S. Department of Energy and the State of Illinois Higher Education Cooperation Act.

A. Pla-Dalmau and A. D. Bross are with Fermi National Accelerator Laboratory, Batavia, IL 60510 USA (e-mail: pla@fnal.gov, bross@fnal.gov).

V. V. Rykalin is with the Northern Illinois Center for Accelerator and Detector Development (NICADD), Northern Illinois University, DeKalb, IL 60115 USA (e-mail: rykalin@fnal.gov). in lower pricing. The estimated price for extruded scintillator ranges between $\$ 5$ and $\$ 8$ per $\mathrm{kg}$.

The Scintillation Detector Development Technical Center at Fermilab has produced extruded plastic scintillator through local extrusion companies for various experiments (D0, STAR, MINOS, K2K) [2]. Initially it was a two-step process where scintillating pellets were first produced (coloring or compounding step) and then used in the final profile extrusion. With MINOS the coloring step was omitted thus becoming a one-step process. In either case, the productions were run in batch mode. The raw materials were pre-mixed and added to the extruder as needed (Fig. 1).

An alternative method is available by using a continuous inline compounding and extrusion process (Fig. 2). The advantage of such technique is the less handling of the raw materials (polystyrene pellets and dopants) with the most direct pathway to the final scintillator product. The challenge in this process is the accurate metering of the raw materials into the extruder, especially the dopant powder. Several extrusion runs have successfully been performed to test this concept and the equipment. Recently, in collaboration with the Northern Illinois Center for Accelerator and Detector Development (NICADD), an extrusion line using this methodology has been installed at Fermilab. A scheme of this extrusion line is depicted in Fig. 3. This extrusion line consists of a Berstorff 40-mm twin-screw extruder (ZE 40A UTS), two K-Tron feeders (one for the polymer pellets and one for the fluorescent dopants) and Conair downstream equipment (vacuum and cooling tanks, belt puller and saw). A compressed-nitrogen dryer from Novatec is utilized to dry and purge the polymer pellets prior to their use. The equipment is owned by NICADD (Northern Illinois University) and jointly operated between Fermilab and NICADD. The facility is aiming to provide $R \& D$ and prototyping of extruded scintillation detectors for nuclear and high energy physics experiments.

\section{EXPERIMENTAL SECTION}

The FNAL/NICADD extrusion line has been operating on a commissioning and R\&D bases since April 2003. Two extrusion dies are currently available. One die yields a $1 \mathrm{~cm}$ by $2 \mathrm{~cm}$ rectangular bar with a 1-mm hole for a wavelength shifting (WLS) fiber. This hole can be collapsed during production to obtain a solid bar. The other die produces a $0.5 \mathrm{~cm}$ by $10 \mathrm{~cm}$ strip with or without 10 (1-mm) holes. The polystyrene (PS) 
pellets used are Dow Styron 663 (W). This is a commercial grade, general-purpose polystyrene without additives. Its price ranges from $\$ 1.2 / \mathrm{kg}$ to $\$ 1.65 / \mathrm{kg}$ based on the quantity purchased. The dopants currently added are 1\% PPO and $0.03 \%$ POPOP available in a pre-mixed form from Curtiss Laboratories (Bensalem, PA). This particular composition costs between $\$ 190 / \mathrm{kg}$ and $\$ 245 / \mathrm{kg}$ depending upon the quantity ordered. The spectroscopic characteristics of this scintillator are shown in Fig. 4. This is a blue-emitting scintillator with an absorption cut-off at approximately $400 \mathrm{~nm}$ (for a 1-cm path length) and an emission maximum at $420 \mathrm{~nm}$.

\section{DISCUSSION}

The first step after installation of the equipment by the vendor was to check if the extrusion line met the required specifications. Most of the emphasis was placed on the equipment performance, extrusion conditions and the size and shape of the extruded material. With this stage completed, the remaining task was to prove that high-quality scintillator could be produced. In order to accomplish this goal, several extrusion runs using the two dies available were carried out. Samples from these runs were saved and tested. Samples of MINOS scintillator were used as reference. With respect to the tolerances of the material produced, special attention was paid to the thickness of the solid scintillator strip prepared with the " $0.5 \mathrm{~cm}$ x $10 \mathrm{~cm}$ " die which was $4.87 \pm 0.03 \mathrm{~mm}$, measured at 20 $\mathrm{cm}$ intervals over a $44-\mathrm{m}$ length.

\section{A. Attenuation Length Measurements}

The FNAL/NICADD extruded scintillator has been tested and compared to the MINOS extruded scintillator that had previously been fully characterized. During the MINOS production, the importance of nitrogen purging was recognized. The oxygen present in the extrusion line and throughout the material causes discoloration of the polymer matrix during processing. This additional absorption will reduce the attenuation length of the scintillator material. Since the FNAL/NICADD extrusion line is not intended to work in a batch mode, the nitrogen purging process will be different from that developed for the MINOS extrusions. However, with the current settings at Fermilab, the attenuation length of the FNAL/NICADD extruded scintillator is the same as that of the MINOS scintillator: $5 \mathrm{~cm}$ for the fast component and $24 \mathrm{~cm}$ for the slow component. Table I lists the data for this measurement. Fig. 5 shows a plot representative of the fitted raw data. For this test 1-m long solid strips (no WLS fiber) were used. A ${ }^{137} \mathrm{Cs}$-source was positioned on the strip at regular intervals from the photo-multiplier tube. The FNAL/NICADD scintillator was checked with and without Tyvek wrapping since the MINOS extrusion has a co-extruded white reflective coating. Differences of the order of 5\% were observed among the samples measured.
T ABLE I

ATTENUATION LENGTH OFEXTRUDED SCINTILLATOR

\begin{tabular}{lcc} 
Sample & Fast Component & Slow Component \\
\hline FNAL/NICADD & $5 \mathrm{~cm}$ & $24 \mathrm{~cm}$ \\
MINOS & $4 \mathrm{~cm}$ & $24 \mathrm{~cm}$ \\
\hline
\end{tabular}

\section{B. Scintillation Light Yield}

The light yield of the FNAL/NICADD extruded scintillator was tested and compared to that of the MINOS scintillator. For this measurement, small samples $(1 \mathrm{~cm} \mathrm{x} 1 \mathrm{~cm} \times 0.6 \mathrm{~cm})$ were cut from the various extrusions. The reflective coating was removed from the MINOS samples. The samples tested presented small variations in size and the final results were normalized to 1-mm scintillator thickness. $\mathrm{A}^{106} \mathrm{Ru}$-source was placed on the extruded scintillator sample that sat on top of the trigger counter. This trigger used a thick scintillator piece to range out the low-energy electrons of the ${ }^{106} \mathrm{Ru}$ spectrum so that it would approximately work in the energy range of a MIP (minimum ionizing particle). The data plotted in Fig. 6 are representative of these measurements. Table II lists the overall results. These numbers were calculated by subtracting the pedestal from the "peak average" and normalizing to 1-mm thickness. These results indicate that the light yield of the bulk material of the FNAL/NICADD extrusions is the same as that of the MINOS extrusions.

TABLE II

SCINTILLATION LIGHT Y IELD OF EXTRUDED SCINTILLATOR

\begin{tabular}{lc} 
Sample & ADC counts \\
\hline 10-hole strip & 3.51 \\
2-hole strip & 3.57 \\
MINOS & 3.48 \\
\hline
\end{tabular}

\section{CONCLUSION}

Even at this early stage of the setup of the FNAL/NICADD extrusion line, the extruded scintillator produced positively compares to the MINOS extruded scintillator. These are two different methods of extruding scintillator with the goal to produce high-quality material. The FNAL/NICADD approach with the in-line continuous extrusion equipment offers the opportunity to see further improvement in attenuation length and light yield as the nitrogen-purging scheme is fully researched for this new equipment configuration.

\section{REFERENCES}

[1] A. Pla-Dalmau, I. Ambats, D. F. Anderson, B. Baller, P. M. Border, B.C. Choudhary et al., "Extruded Plastic Scintillator for the MINOS Calorimeters", Frascati Physics Series vol. XXI, pp. 513-522, 2000.

[2] A. Pla-Dalmau, A.D. Bross and K.L. Mellott, "Low-Cost Extruded Plastic Scintillator", Nucl. Instr. Meth. Vol. A466, pp. 482-491, 2001. 


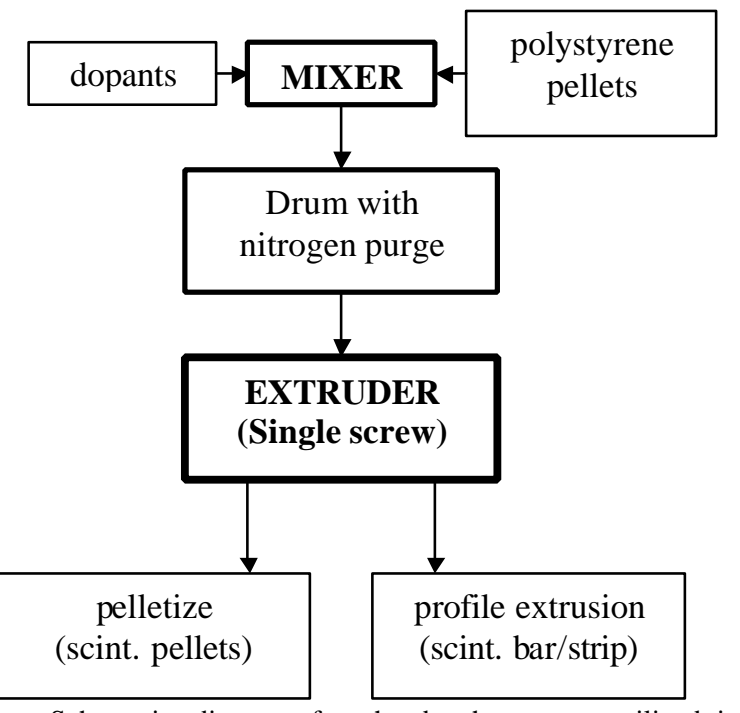

Fig. 1. Schematic diagram for the batch process utilized in the manufacture of extruded plastic scintillator.

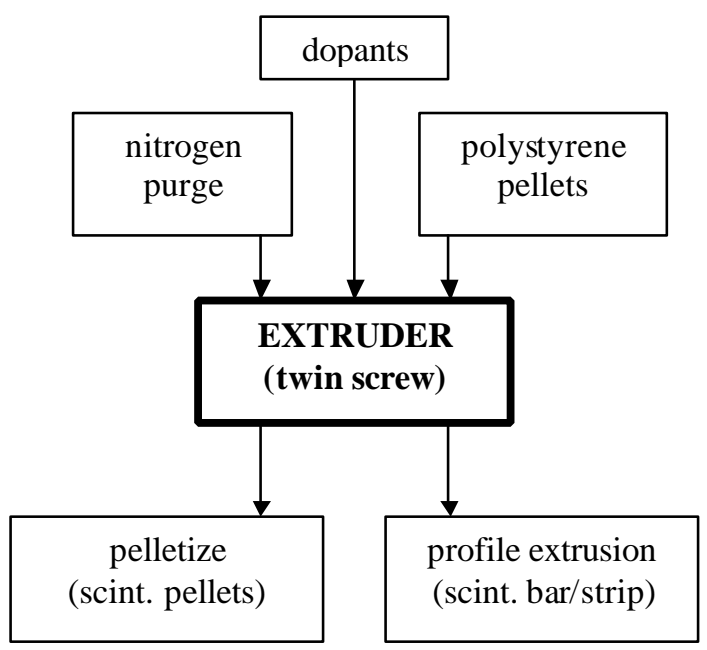

Fig. 2. Schematic diagram for the continuous process utilized in the preparation of extruded plastic scintillator.

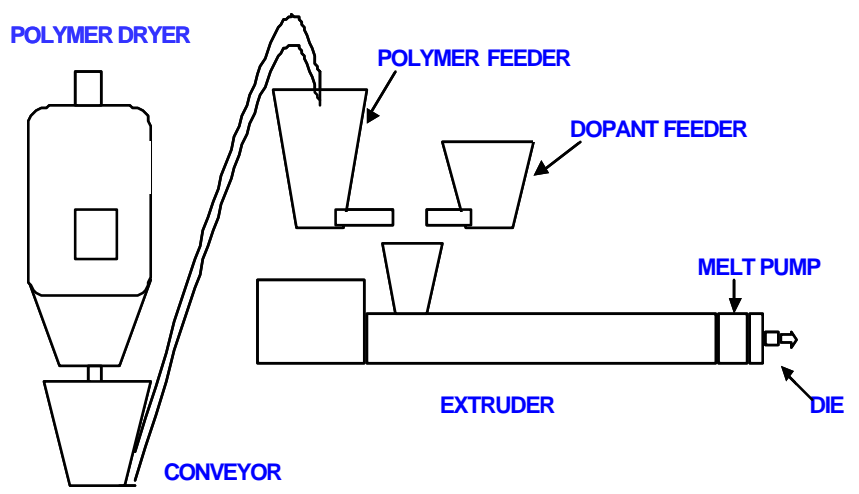

Fig. 3. Schematic diagram of the FNAL/NICADD continuous extrusion line for plastic scintillator.

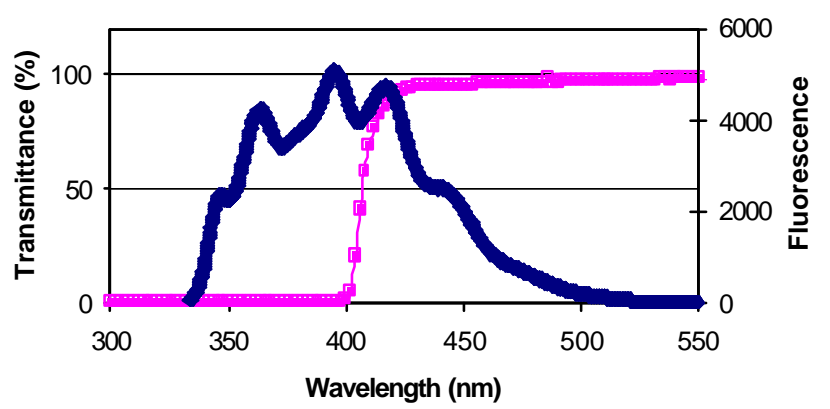

Fig. 4. Transmittance and fluorescence spectra of FNAL/NICADD extruded scintillator $(1 \% \mathrm{PPO}+0.03 \%$ POPOP $)$. Sample is $1-\mathrm{cm}$ thick disk. Reference for transmittance measurement is a polystyrene disk of the same thickness. Fluorescence measurement uses $310 \mathrm{~nm}$ excitation wavelength of surface closest to the detector; both PPO and POPOP bands are observed.

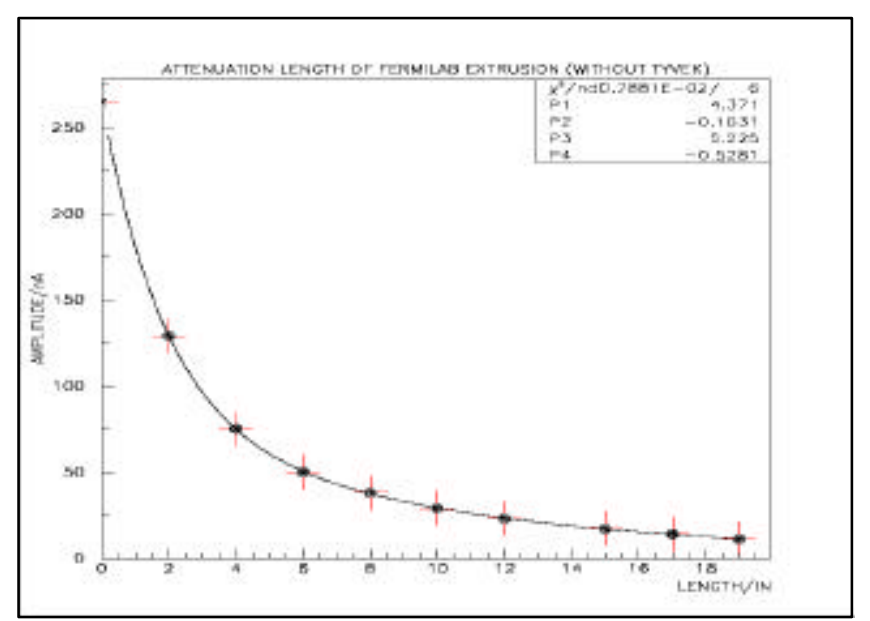

Fig. 5. Plot representative of the attenuation length measurement performed using a ${ }^{137} \mathrm{Cs}$ radioactive source on $1 \mathrm{~m}$ long solid bars of FNAL/NICADD extruded scintillator with a cross-section of $1 \mathrm{~cm}$ by 2 $\mathrm{cm}$ (no WLS fiber).

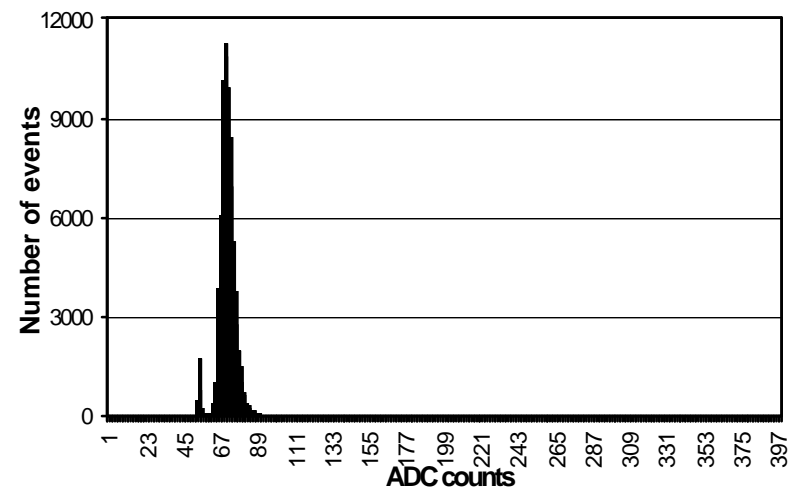

Fig. 6. Light yield measurement data for extruded scintillator using a ${ }^{106} \mathrm{Ru}$ radioactive source and triggering on the high-energy electrons. The sample size is $1 \mathrm{~cm} \times 1 \mathrm{~cm} \times 0.6 \mathrm{~cm}$ cut from a 10-hole strip. 